\title{
Civilisations
}

Revue internationale d'anthropologie et de sciences

humaines

$48 \mid 2001$

La question de l'islam et de l'Etat à l'aube du XXIe siècle

\section{Modernisation de l'islam ou islamisation de la modernité ?}

Les minorités musulmanes d'Europe et l'enjeu du pluralisme

Jocelyne Cesari

\section{(2) OpenEdition}

\section{Journals}

Édition électronique

URL : http://journals.openedition.org/civilisations/3470

DOI : $10.4000 /$ civilisations. 3470

ISSN : 2032-0442

Éditeur

Institut de sociologie de l'Université Libre de Bruxelles

Édition imprimée

Date de publication : 31 août 2001

Pagination : Page 139-144

ISSN : 0009-8140

Référence électronique

Jocelyne Cesari, « Modernisation de l'islam ou islamisation de la modernité ? », Civilisations [En ligne], 48 | 2001, mis en ligne le 01 août 2004, consulté le 19 avril 2019. URL : http://

journals.openedition.org/civilisations/3470 ; DOI : 10.4000/civilisations.3470

(C) Tous droits réservés 


\title{
MODERNISATION DE L'ISLAM OU ISLAMISATION DE LA MODERNITE ? LES MINORITES MUSULMANES D'EUROPE ET L'ENJEU DU PLURALISME
}

\author{
Jocelyne CESARI
}

La question du rapport entre islam et modernité est aussi ancienne que la confrontation entre l'Europe des Lumières et le monde arabo-musulman. L'entrée de Bonaparte au Caire en 1798 a constitué le moment symbolique de cette rencontre entre l'Occident porteur de modernité et les peuples de l'Orient musulman alors sous domination ottomane. Cette rencontre fut un choc pour le monde musulman : un occident militairement et scientifiquement puissant se révélait à lui qu'il ne pouvait continuer d'identifier au vieil ennemi chrétien parce que cen'était pas le christianisme mais l'Aufklärung qui inspirait son action. Cette confrontation allait susciter du côté musulman un examen de conscience et un souci d'aggorniamento. Cette réflexion sur la modernité se fera sous le mode non pas de la rupture mais du recours à la tradition non pas du progrès mais de la renaissance. En 1880 , le premier courant réformiste politico-religieux, la salafiyya illustrée par Djamal-al-din-al Afghani et Muhammad Abduh, fondait sa vision réformiste sur des prémisses opposées à celles des Lumières : aller vers plus et non pas moins de religion. La décadence était considérée comme une conséquence du despotisme des gouvernants et d'une interprétation superstitieuse de la religion. La supériorité de l'Occident était perçue comme uniquement matérielle et technique, le vrai progrès ne pouvant être que spirituel et éthique. La question de la rationalisation des données de la foi a été au cour du débat et a donné lieu à une série d'exercices théoriques afin de démontrer qu'il n'y a pas d'incompatibilité entre l'islam et les techniques ni même avec les principes politiques de l'Occident. Ce débat plus que jamais d'actualité connaît de nouveaux rebondissements avec l'installation de l'islam en Europe.

\section{ISLAMISATION DE LA MODERNITÉ}

La salafiyya a constitué le premier courant décisif pour inscrire la modernisation dans un cadre de référence islamique. Face aux défis de la modernité, les tenants de ce mouvement plaidaient un recours à la tradition musulmane qui détenaient selon eux, les ressources nécessaires pour une réactualisation de la foi des Anciens (Salaf) et ce en rejetant le poids des superstitions et des compromissions qui ont dénaturé la vraie nature du message révélé. Contrairement à l'élite qui voyait la modernité comme absorption et transfert de techniques, 
les réformateurs musulmans ont plaidé contre la rupture entre passé et présent en conciliant acquis de la modernité et patrimoine musulman. À cet égard, les réformistes n'ont cessé de prôner l'ijtihad (effort d'interprétation) afin de lutter contre des formes de tradition qui figeaient l'islam en le rendant inadapté aux situations de nouveauté tant dans le domaine social que dans les domaines culturels ou politiques.

Contrairement à certaines idées reçues, dans un premier temps, le traumatisme de l'impact occidental ne s'est pas accompagné d'un clivage entre occidentalistes et anti-occidentalistes mais par un intense besoin de réforme de l'islam. Ce besoin a réactivé avec une acuité nouvelle un clivage fort ancien et connu entre islam lettré et islam populaire en donnant la prééminence et la priorité au premier (Gellner, 1992). L'islam lettré est celui des universitaires urbanisés issus de la bourgeoisie marchande, reflétant les valeurs de ces groupes : ordre, sobriété, étude avec une aversion très nette pour toute forme $d$ 'hybris ou d'émotion excessive en religion. En revanche, l'islam populaire est quant à lui empreint de rites magiques, d'extase, peuplés de médiateurs tels que les marabouts ou les chefs de confrérie. La Salafiyya a constitué un moment décisif de victoire de l'islam lettré et urbanisé. Elle est apparue comme une tentative d'ancrage, dans le socle de la culture locale, des acquis au départ exogènes de la modernité. Elle a été la matrice de deux courants antagonistes qui aujourd'hui déchirent le monde musulman : l'un occidentaliste séculier, l'autre identitaire islamique.

Entre les deux guerres mondiales, l'équilibre s'est rompu au profit du courant occidentaliste, entrainant l'arrêt de l'œuvre culturelle des réformistes. La suprématie accordée aux Lumières occidentales s'est traduite par une multiplication d'instituts et d'écoles destinés à former les futurs cadres administratifs et militaires selon les idées «importées». Un nouveau type d'élites a émergé, lesquelles au nom de la modernisation politique et des normes empruntées au modèle $\mathrm{du}$ colonisateur sont ensuite devenues entre les deux guerres les principaux artisans et promoteurs du nationalisme et du panarabisme. Cette brisure dans le mouvement initial de la réforme explique en partie le basculement d'une partie de la pensée politique islamique dans le radicalisme et l'antimodernisme.

Ce débat loin d'être achevé connaît aujourd'hui de nouveaux rebondissements avec l'émergence d'un islam d'Occident.

\section{... OU MODERNISATION DE L'ISLAM?}

L'entrée de l'islam dans les espaces démocratiques sécularisés a eu comme conséquence de faire du choix individuel l'élément central du rapport à la religion. Cette évolution est une véritable révolution culturelle dans la mesure où les familles proviennent de pays où l' islam est religion de la majorité de la population, quand ce n'est pas religion d'État. C'est ainsi que pour leurs parents, l'islam est bien plus qu'une religion engageant des actes de piété ou des rituels mais correspond davantage à un référent collectif qui définit les rapports sociaux mais aussi l'identité nationale et politique. Dans les pays d'origine, particulièrement au Maghreb, l'islam est un élément du ciment social et ne se réduit pas à la religiosité. Être musulman en Europe revient à faire sortir le lien à l'islam de son évidence, de son statut de donné communautaire, culturel ou social, pour le faire entrer dans la sphère des choix individuels et donc du questionnement.

Cette individualisation est rendue possible par la décomposition de la communauté ethnique d'origine. L'exil et l'enracinement ont des conséquences irrémédiables sur les 
composantes culturelles du groupe d'origine. L'absence d'autorités religieuses légitimes favorise également l'individualisation ${ }^{1}$.

Cet affaiblissement de l'emprise du groupe d'origine rend possible deux lignes d'évolution possible : soit la sortie du groupe et de l'islam, soit la quête d'un islam authentique, purifié des «écorces» des traditions et des cultures. La première option reste pour l'instant quasiment inexistante en raison du rythme d'installation de cette religion dans un espace culturel sécularisé. En effet, l'enracinement de l'islam est récent et doit être distingué de l'installation, plus ancienne, des musulmans : il aura fallu plus d'une génération pour que les immigrés musulmans acceptent de considérer la France comme «terre d'islam» et c'est seulement leurs enfants et surtout leurs petits enfants qui tentent de concilier depuis quelques décennies attachement à une tradition importée et ancrage dans le terreau culturel français. Cette conciliation implique sécularisation, agnosticisme mais pas encore véritablement athéisme. En effet, la sortie complète de l' islam semble pour l'instant impossible, notamment pour les jeunes gens d'origine maghrébine, en raison du syndrome postcolonial et du «double lien» qu'il entretient. Mais nul ne peut préjuger des évolutions d'une génération à l'autre.

La seconde option, réformiste, emprunte deux voies divergentes : soit un rapport sécularisé à l'islam qui tend à relativiser au maximum les injonctions de la tradition ou au contraire un fondamentalisme qui s'inscrit dans un respect exigeant de la tradition dans sa globalité.

\section{Les citoyens de confession islamique}

L'islam sécularisé concerne les personnes, pour leur grande part nées ou scolarisées en France, qui, sans toujours se conformer aux règles du culte, considèrent la référence islamique comme une partie de leur héritage familial et culturel. Cette immense majorité silencieuse est en train de positionner dans la plus grande discrétion, pour ne pas parler d'indifférence, la référence islamique dans la sphère du privé, voire de l'intime. Pourtant elle ne suscite paradoxalement aucun intérêt médiatique alors que sa pratique constitue en soi une véritable révolution culturelle.

Sans abandonner l'identification à l'islam, ces musulmans sécularisés l'envisagent comme source de valeurs donnant un sens à leur vie sans pour autant impliquer chez eux un passage à la pratique. Ce processus de «mobilisation-subjectivation» des valeurs dégage le message islamique des contenus et des normes établies dans la tradition. Ainsi le sujet marque-t-il son individualité par rapport au groupe et se place-t-il comme médiateur entre le contenu du code et son application. Les formes ainsi dégagées de leur détermination primitive et contraignante deviennent accueillantes à son inventivité. Ceci n'est pas sans rappeler les nouvelles formes de religiosité dans les sociétés modernes où le croyant n'obéit plus aux normes transmises par l'institution ou la tradition mais choisit les biens du salut en fonction de son individualité. La dimension collective de l'appartenance islamique est ainsi consentie et régulée par la logique individuelle, ce qui constitue des manières inédites et tout à fait originales de rapport au référent islam qui n'en sont qu'à leurs prémices.

Ce processus consacre la nouveauté d'une réappropriation de la formule religieuse. Être musulman ne va pas de soi dans un environnement au mieux indifférent au pire hostile. Alors que les parents ont vécu dans un environnement où il y avait une relative cohérence entre identités religieuse, sociale et nationale, leurs enfants découvrent au mieux l'écart au pire la 
contradiction entre identité individuelle, identité collective, identité nationale, et qui plus est dans un climat d'incompréhension voire même de franche hostilité.

Ces transformations impliquent un rapport émotionnel et culturel à l'islam. Elles concernent $80 \%$ des nouvelles générations éduquées en France. Dans cette mouvance majoritaire, l'identification à l'islam permet seulement de se rattacher à une tradition sans pour autant s'accompagner d'une véritable pratique religieuse. De même qu'il existe un catholicisme confessant et séculier (Lambert et Michelat, 1992), nous assistons donc à l'émergence d'un islam confessant et séculier ${ }^{2}$.

Plusieurs modalités se dégagent dans ce courant de l'islam confessant.

La première renvoie à une relation privatisée à Dieu. Toutes les injonctions de l'islam font l'objet d'accommodations, d'adaptations en fonction de la situation personnelle du croyant. Il est très courant de rencontrer des jeunes des deux sexes qui «shahadent» ${ }^{3}$, respectent les interdits alimentaires, font la prière de temps en temps, jeûnent durant le mois de ramadan mais refusent d'apparaître comme musulmans dans les relations sociales. En général, ce type de comportements nécessite une certaine connaissance de la tradition afin de mieux pondérer ses choix.

Une deuxième modalité, que l'on pourrait qualifier d'islam identitaire revient à concilier le maximum d'autonomie individuelle avec la croyance en une transcendance plus ou moins définie que l'on gère avec les contraintes de son époque, au minimum à travers les grands rites de passage : circoncision, mariage, enterrement. Ce sont eux qui se définiront comme «croyants non pratiquants». Bon nombre de ces croyants non véritablement pratiquants ne rejettent pas l'islam ethnique transmis par les parents qui les conforte dans un rapport festif et traditionnel à l'islam.

De plus, ce deuxième sous-groupe de l'islam identitaire se subdivise entre ceux qui peuvent être qualifiés «d'orthodoxes non pratiquants» et ceux qui évoluent vers une sortie de l'islam. Les premiers, majoritaires, font du respect de la tradition une norme et considèrent simplement qu'ils ne sont pas à la hauteur ou n'ont pas les moyens en raison de l'environnement et des contraintes de la société dans laquelle ils vivent, de pouvoir respecter cette tradition. Ils se recrutent plutôt dans les milieux populaires ou les petites classes moyennes issus de l'immigration. Ils montrent peu de connaissance de la tradition islamique et des prescriptions rituelles qui en découlent. La plupart n'ont reçu aucun enseignement coranique, que ce soit dans ou hors de la famille (écoles coraniques, etc...).

L'islam renvoie ici à une préservation de la fidélité au groupe d'origine sans qu'il engage véritablement un sentiment de croyance ou de piété. Les personnes issues de la bourgeoisie maghrébine, notamment algérienne et tunisienne, sont très représentatives de cette manière de vivre l'islam.

Plus généralement, pour les tenants de l'islam identitaire, ce terme est associé à des séquences de la vie familiale, notamment à l'occasion de la célébration des fêtes (telle l'Aid el Kebir), impliquant une rupture avec l'espace et le temps environnants. Ensuite, ce terme est aussi associé au respect dû aux croyances et pratiques parentales sans que cela implique un même conformisme de la part des sujets concernés. L'islam est alors conçu comme un héritage culturel inscrit dans des traditions et des comportements familiaux les rattachant au groupe arabo-musulman. Il est en fait un «marqueur» de la filiation qui le positionne plus dans le registre de la culture que de la religion. 


\section{Les intégralistes: une nouvelle célébration des origines}

Un second groupe, largement minoritaire, se définit quant à lui, par une grande exigence dans le respect des prescriptions islamiques. La religion est en ce cas investie comme une orthopraxis. L'entrée en islam favorise une prise sur le réel ou à tout le moins donne des repères et des balises. Le monde est balisé en «pur» et «impur» et tous les actes peuvent être classés en fonction d'un degré de licite ou d'illicite. Tous les témoignages décrivent cette mise en conformité des comportements en fonction des prescriptions : qu'il s'agisse de l'alimentation du vêtement, des actes rituels.

L'islam en tant que synonyme d'éducation est également associé aux idées de culture et de formation. Tous témoignent d'une recherche personnelle qui prend la forme d'un apprentissage de l'arabe classique que la plupart, enfants de la cité, ne connaissent pas. Ils se lancent également dans une approche des textes révélés, une lecture d'ouvrages généraux sur les fondements de la tradition musulmane, dans leur grande majorité traduits en français. Les principaux livres accessibles en langue française dans presque toutes les librairies, concernent la description des piliers de l'islam et des prescriptions dans différents domaines sociaux, économique, culturel, éducation etc... la biographie du Prophète et les vies exemplaires de certains Compagnons illustres, la question du statut de la femme ou encore des rapports entre islam et science.

Émerge une conception de la religion, source de morale et éducative, qui exalte la logique du choix individuel (libre-arbitre) et introduit une rupture avec l'ethnicisation de la religion. Tous mettent en avant une logique individuelle de décisions et de choix qui s'inscrit bien dans cette subjectivation croissante de l'appartenance religieuse. Il ne suffit pas de croire et pratiquer parce que l'on est né dans telle ou tradition croyante mais d'exprimer son individualité en faisant le choix d'être un pratiquant et en redonnant un sens personnel au message révélé. En résulte alors une mise à distance avec la famille, justifiée par le fait que les parents ne comprennent pas ce qu'est le «véritable» islam, qu'ils n'ont pas de véritable connaissance et agissent sous l'influence des coutumes et de superstitions liées à leur culture.

A partir de cette conception commune de la religion, les trajectoires diffèrent. L'entrée dans l'islam comme acte «rédempteur» est bien sûr très affirmé mais pas exclusif (Cesari, $1998)^{4}$.

Cette recherche d'un islam universel, savant et régi par la logique individuelle met en évidence l'expérience tout à fait inédite de reconstruction dans le contexte français d'une lignée croyante et souligne en même temps l'extrême difficulté de cette opération qui nécessite des ruptures souvent difficiles avec le milieu familial ainsi que des adaptations du contenu de la Tradition musulmane au contexte de minorité musulmane. A cet égard, les référents et les décisionnaires qui pourraient servir de garde-fou sont extrêmement rares. Tous nous ont décrit un parcours fait de tâtonnements, d'incompréhensions voire de rigidités qu'ils essaient de combattre en particulier au début de toute entrée dans l'islam, selon le schéma classique du surinvestissement du néophyte. C'estlà quel'action associative joue un rôle positifd'encadrement et de maîtrise de la pratique afin d'éviter toute surenchère et toute velléité d'ostracisme ou de rejet de l'autre, musulman ou pas, au nom de l'islam.

Ces acteurs minoritaires sont engagés dans un processus d'édification de nouvelles communautés sur la base du lien islamique et de l'adhésion volontaire et réfléchie aux prescriptions religieuses. A partir de ces choix communs, se développe un sentiment de 
fraternité qui fait de l'appellation «frère» ou «sœur» bien plus qu'une simple convention, la reconnaissance d'un même choix, librement consenti. A cet égard, la dimension émotionnelle et affective est également significative et détermine bien souvent les modalités d'entrée dans l'islam. Certains, parmi les plus jeunes, ont en effet mentionné qu'ils sont devenus pratiquants au cours du mois de ramadan qui constitue une période très privilégiée de la cohésion et de la solidarité communautaires. Toutes les formes de réunions, de rencontres, d'apprentissage en commun entretiennent cette émotion partagée entre jeunes. C'est pourquoi les associations multiplient ce type d'activités dans une gamme très large qui va du festif au culturel.

Ces trajectoires d'islamisation, bien que pourl'instant minoritaires, éclairent les profondes mutations qui affectent de manière générale une partie de la jeunesse des classes populaires et de manière spécifique celle d'origine immigrée. En effet, cette islamisation qui procède par recréation de la lignée croyante, répond à l'effritement généralisé du lien social atteignant non seulement les institutions mais aussi la famille.. Elle s'inscrit dans une diversification des identités musulmanes qui témoignent de leur entrée de plain pied dans le pluralisme politique et culturel.

\section{BIBLIOGRAPHIE}

Cesari, J.1998. Musulmans et Républicains: les jeunes, l'islam et la France, Bruxelles, Complexe, 1998.

Gellner, E. 1992. Postmodernism, Reason and Religion, Londres, Routledge.

Lambert, Y et Guy Michelat (éd.) 1992, Crépuscule des religions chez les jeunes? Jeunes et Religions en France, Paris, L'Harmattan.

\section{NOTES}

1 Dans la diaspora, le musulman expérimente véritablement l'absence de médiation entre le texte révélé et sa propre singularité. Non pas qu'il n'y ait pas d'autorités islamiques en France mais elles ne sont pas adaptées aux aspirations de cet islam minoritaire. En dépit de la formule toujours rappelée que «le musulman n'a de compte à rendre qu'à Dieu», que d'aucuns utilisent à bon escient pour discréditer le leadership d'éventuels concurrents, il faut néanmoins rappeler qu'un corps de spécialistes a toujours exercé une double fonction d'exégèse et de jurisprudence des textes révélés. Ces docteurs de la Loi (ulemas) doivent bien évidemment être distingués des imâms, terme qui dans la tradition musulmane n'est pas un titre mais désigne simplement celui qui dirige la prière collective. L'absence de telles figures d'autorité dans l'espace islamique français devient une véritable difficulté en raison de la condition minoritaire dans un contexte démocratique qui n'a pas été jusqu'à présent formalisée par la tradition musulmane.

2 Les résultats d'un sondage SOFRES-Le Nouvel Observateur révèlent ce courant majoritaire : 49\% des personnes interrogées considèrent l'islam comme une affaire privée, $87 \%$ déclarent l'islam compatible avec la République et $57 \%$ refusent l'éventualité d'un parti musulman. Voir Le Nouvel Observateur du 15 au 21 janvier 1998.

3 C'est-à-dire récitent quotidiennement la Profession de foi : «il n'y a de Dieu que Dieu et Muhammad est son Prophète».

4 Trois types différents d'acteurs se dégagent : l'enfant des cités, le grimpeur social et l'intellectuel transplanté. 